\title{
Polarization Dependence of Area Scanning Ultrafast Laser Machining
}

\author{
Mindaugas Gecevičius, Martynas Beresna, Jingyu Zhang, Peter G. Kazansky \\ Optoelectronics Research Centre, University of Southampton, SO17 1BJ, United Kingdom \\ mg2e10@orc.soton.ac.uk
}

\begin{abstract}
Polarization dependence of laser machining by area scanning is observed. This general phenomenon is explained by anisotropies of stress induced in material and spatiotemporal distortions in ulrashort pulse. The control of phenomenon is demonstrated.
\end{abstract}

OCIS codes (140.3390) Laser materials processing; (260.7120) Ultrafast phenomena; (260.1440) Birefringence

Recently fabrication of polarization sensitive elements (such as polarization diffraction gratings or converters for radially polarized light) fabricated in fused silica by area scanning with femtosecond laser were demonstrated [1]. These elements are based on laser induced nanogratings which are responsible for form birefringence [2]. The induced retardance and the slow axis are controlled by laser exposure and laser light polarization respectively. As the direction of birefringence slow axis has to be modulated, the angle between laser polarization and writing direction cannot be fixed during the fabrication procedure. For efficient device performance, it is of paramount importance that retardance would not depend on laser light polarization. Difference in modification between $0^{\circ}$ and $90^{\circ}$ angle of polarization with respect to writing direction attributed to front of the line boundary conditions is known in laser micromachining [3]. Here we report observation of fundamentally new two types of writing polarization dependence revealed in area scanning and not related to the boundary conditions.

Retardance dependence on polarization is quite strong (Fig. 1 (a)). As difference in modification between $0^{\circ}$ and $90^{\circ}$ angle of polarization with respect to writing direction could be explained by the effect of the boundary condition factor, difference between $45^{\circ}$ and $-45^{\circ}$ is unexpected and counterintuitive. Moreover, in some cases (like in example shown in Fig. 1 (a)) this difference is even stronger than for $0^{\circ}$ and $90^{\circ}$ angle, so it is important to understand the causes of this asymmetry and how to reduce its impact.

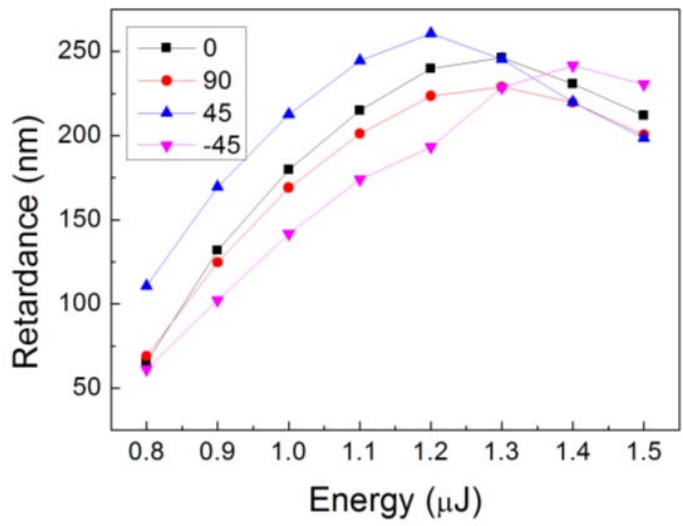

(a)
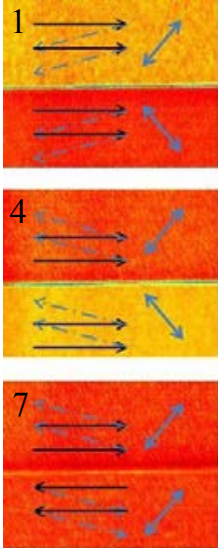

(a) Retardance dependence on pulse energy for four different polarizations of laser light (at $1.2 \mu \mathrm{J}$ curves start bending because retardance measurement system Abrio cannot measure values more than $\sim 250 \mathrm{~nm}$ ). (b) Microscopic retardance images of structures written with various combinations of polarization direction, line writing direction and area filling direction. Arrows on each structure indicate writing polarization direction and fabrication algorithm.

The experiments were done with femtosecond laser system PHAROS (LightConversion Ltd.) with pulse duration stretched from $300 \mathrm{fs}$ to $800 \mathrm{fs}$. Beam was focused with an aspheric lens $(\mathrm{NA}=0.16)$. Separation between lines was $1 \mu \mathrm{m}$. The samples were inspected with a quantitative birefringence measurement system Abrio (CRi Inc.).

The birefringence analysis of laser written lines revealed arrow-shape-like stress, which is pointing towards scanning direction. This leads to hypothesis, that the strength of structure might depend on the polarization angle with respect to the stress induced by previous line. In this case the effect should depend on line writing and area filling directions. It should also disappear by increasing distance between lines. In order to prove it, several squares were written with various combinations of polarization direction, line writing direction and area filling direction (Fig. 1 (b)).

Clear correlation of strength of retardance and angle between laser polarization and stress induced by previous line was observed. Retardance was much stronger for polarization perpendicular to induced stress than 
for parallel. This shows that in some cases effect caused by different writing polarizations can be compensated by choosing correct scanning algorithm (the third and the sixth structures in Fig. 1 (b)).

As we assumed that this affect is caused by previously written line, it should disappear by increasing separation between lines. The second and the third structures shown in Fig. 1 (b) were written with different separation between lines (from $0.5 \mu \mathrm{m}$ to $3 \mu \mathrm{m}$ ). The structure which had retardance value dependence on polarization under small separation between lines (the second in Fig. 1 (b)) became the same for both polarizations (Fig. 1 (a)).

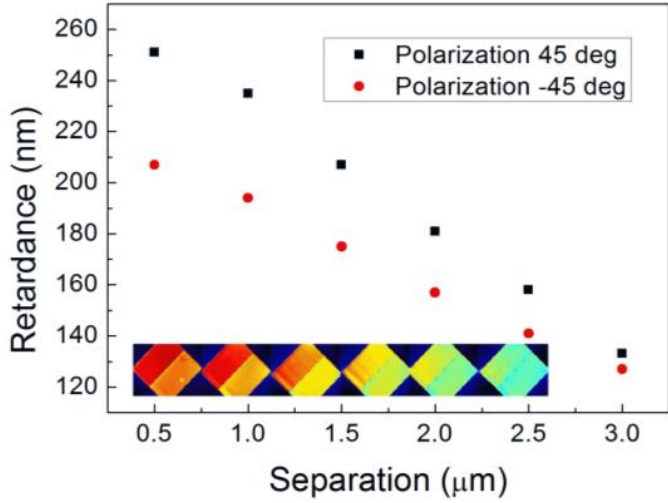

(a)

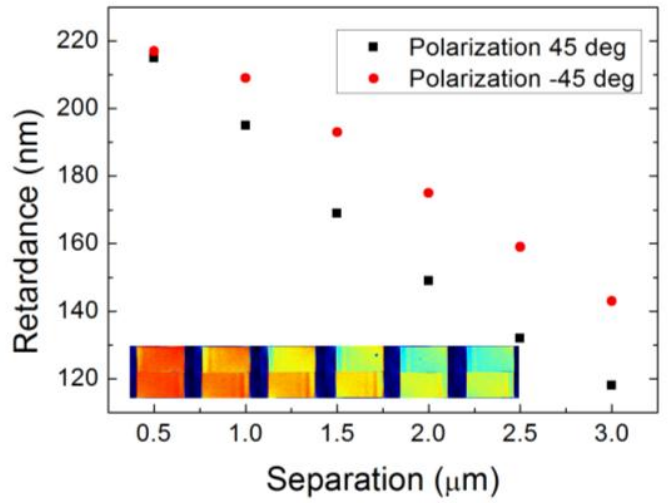

(b)

Fig. 2. Retardance dependence on separation distance between lines for two polarizations. Structure fabrication algorithm: (a) the second structure in Fig. 1 (b); (b) the third structure in Fig. 1 (b).

On the other hand, unexpected result was obtained in the structure (the third in Fig. 1 (b)) which did not have retardance dependence on polarization at small separations. At larger line separation the another type of dependence appeared (Fig. 2 (b)). This indicates that polarization-stress interaction is not the only mechanism causing the retardance strength dependence on polarization. Indeed, strength of modification can also depend on the angle between pulse front tilt and polarization [4]. Pulse front tilt measurements showed that 800 fs pulse had a tilt in the direction of about $45^{\circ}$. If the pulse front tilt is responsible for asymmetry for $-45^{\circ}$ and $45^{\circ}$ polarization angles then the latter should disappear when the structure is written along the pulse front tilt direction.

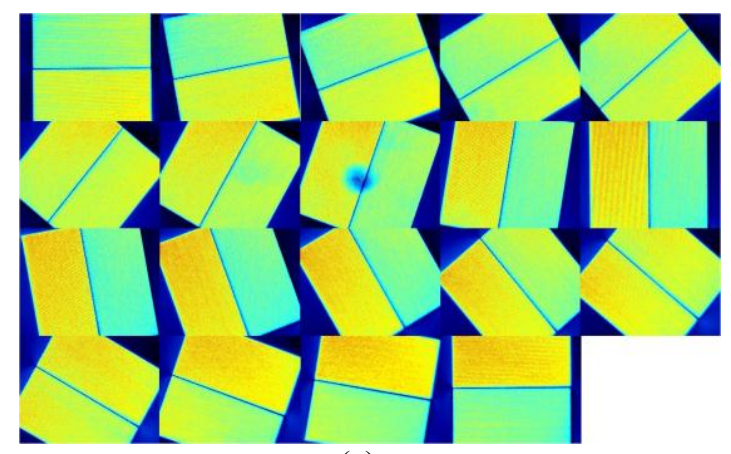

(a)

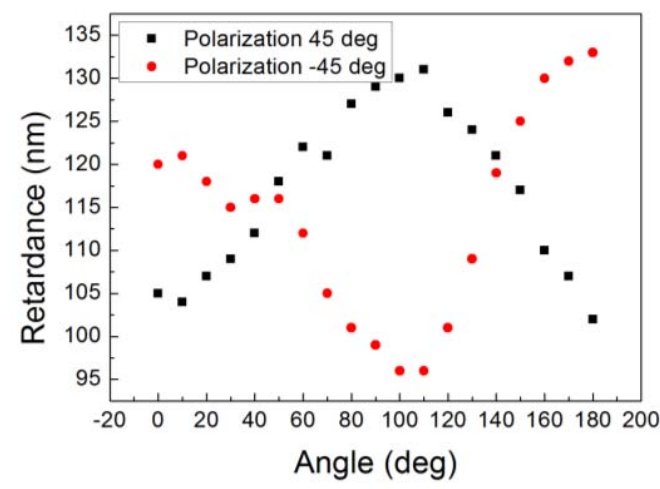

(b)

Fig. 3. Retardance variation for two writing polarizations when structure was written is angles. Separation between lines was $3 \mu \mathrm{m}$, fabrication algorithm as in the third structure in Fig. 1 (b).

The structure (the third in Fig. 1 (b)) with $3 \mu \mathrm{m}$ separation between lines was written at different angles. Polarization was rotated each time to keep the same $45^{\circ}$ angle with writing direction (Fig. 3 (a)). Big difference in retardance was observed at $0^{\circ}$ and $90^{\circ}$ and it disappeared at about $45^{\circ}$. This result correlates well with the pulse front tilt direction.

\section{References}

[1] M. Beresna, M. Gecevičius, and P. G. Kazansky, "Polarization sensitive elements fabricated by femtosecond laser nanostructuring of glass [ Invited ]," Optical Materials 1, 10117-10124 (2011).

[2] E. Bricchi, B. G. Klappauf, and P. G. Kazansky, "Form birefringence and negative index change created by femtosecond direct writing in transparent materials," Optics letters 29, 119-21 (2004).

[3] K. Venkatakrishnan, B. Tan, P. Stanley, and N. R. Sivakumar, "The effect of polarization on ultrashort pulsed laser ablation of thin metal films," Journal of Applied Physics 92, 1604 (2002).

[4] P. G. Kazansky, Y. Shimotsuma, M. Sakakura, M. Beresna, M. Gecevičius, Y. Svirko, S. Akturk, J. Qiu, K. Miura, and K. Hirao, "Photosensitivity control of an isotropic medium through polarization of light pulses with tilted intensity front.," Optics express 19, 2065764 (2011). 